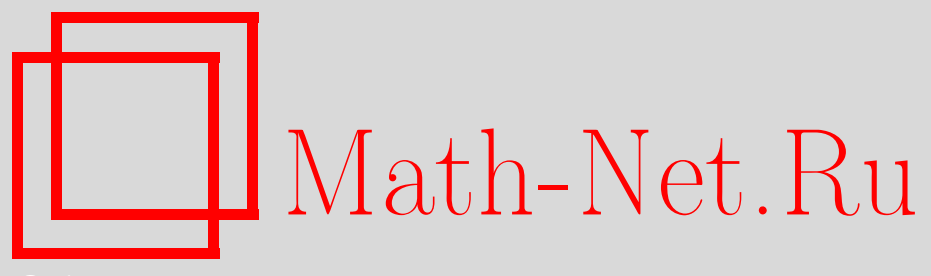

М. Е. Широков, О квантовой пропускной способности при нулевой ошибке, УМН, 2015, том 70, выпуск 1, 187188

DOI: https://doi.org/10.4213/rm9642

Использование Общероссийского математического портала Math-Net.Ru подразумевает, что вы прочитали и согласны с пользовательским соглашением http: //www . mathnet.ru/rus/agreement

Параметры загрузки:

IP: 34.229 .108 .108

26 апреля 2023 г., 13:19:42

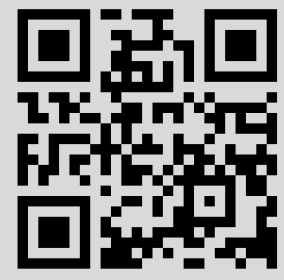




\section{О квантовой пропускной способности при нулевой ошибке}

\section{М. Е. Широков}

Понятие пропускной способности канала связи при нулевой ошибке появилось в классических трудах Шеннона; существенное продвижение в его изучении связано с работами Ловача. Квантовое обобщение этого понятия и связанного с ним понятия некоммутативного графа смежности дано в [1], [2]. В настоящей заметке построено семейство квантовых каналов $\Phi_{n}$ с положительной (асимптотической) квантовой пропускной способностью при нулевой ошибке, для которых соответствующая $n$-шаговая пропускная способность равна нулю. Этот эффект является новым проявлением недавно открытого феномена "суперактивации" пропускных способностей квантового канала при блочном кодировании [3]-[5].

Квантовым каналом называется линейное вполне положительное сохраняющее след отображение $\Phi: \mathfrak{M}\left(\mathscr{H}_{A}\right) \rightarrow \mathfrak{M}\left(\mathscr{H}_{B}\right)$, где $\mathfrak{M}\left(\mathscr{H}_{X}\right)$ - алгебра всех операторов в конечномерном унитарном пространстве $\mathscr{H}_{X}$.

ОПределение (см. [6; гл. 9]). Квантовый канал $\Phi: \mathfrak{M}\left(\mathscr{H}_{A}\right) \rightarrow \mathfrak{M}\left(\mathscr{H}_{B}\right)$ обратим на подпространстве $\mathscr{H}_{0} \subset \mathscr{H}_{A}$, если существует квантовый канал $\Psi: \mathfrak{M}\left(\mathscr{H}_{B}\right) \rightarrow \mathfrak{M}\left(\mathscr{H}_{A}\right)$ такой, что $\Psi(\Phi(\rho))=\rho$ для любого состояния $\rho$ с носителем в $\mathscr{H}_{0}$.

Сужение канала $\Phi$ на подалгебру $\mathfrak{M}\left(\mathscr{H}_{0}\right)$ будем называть идеалъным подканалом канала $\Phi$. Именно идеальные подканалы осуществляют безошибочную передачу квантовых состояний при однократном использовании канала $\Phi$. Если же используется блочное кодирование для $n$ копий канала $\Phi$, то безошибочная передача квантовых состояний осуществляется идеальными подканалами канала $\Phi^{\otimes n}$.

В данной заметке показано, как для любого $n \in \mathbb{N}$ явно построить такой квантовый канал $\Phi_{n}$, что у канала $\Phi_{n}^{\otimes n}$ (а значит, и у каналов $\Phi_{n}^{\otimes k}, k<n$ ) нет идеальных подканалов, но при некотором $m>n$ у канала $\Phi_{n}^{\otimes m}$ появляется идеальный подканал. Это означает, что, используя не более $n$ копий канала $\Phi_{n}$, нельзя безошибочно передать никаких квантовых состояний, но такая передача становится возможной, если число используемых копий данного канала не меньше $m$, причем это число $m$ можно явно вычислить по заданному $n$.

Для фиксированного $\theta \in(-\pi, \pi]$ рассмотрим подпространство

$$
\mathfrak{L}_{\theta}=\left\{\left[\begin{array}{cccc}
a & b & \gamma c & d \\
b & a & d & \bar{\gamma} c \\
\bar{\gamma} c & d & a & b \\
d & \gamma c & b & a
\end{array}\right], a, b, c, d \in \mathbb{C}, \gamma=\exp \left(\frac{\mathrm{i}}{2} \theta\right)\right\}
$$

алгебры всех $(4 \times 4)$-матриц, которое можно считать деформацией максимальной коммутативной *-подалгебры $\mathfrak{L}_{0}$ (ср. [7]). Поскольку $\mathfrak{L}_{\theta}$ симметрично $\left(\mathfrak{L}_{\theta}^{*}=\mathfrak{L}_{\theta}\right)$, содержит единичную матрицу и $\operatorname{dim} \mathfrak{L}_{\theta}=4$, нетрудно явно построить квантовые каналы с $\mathscr{H}_{A}=\mathbb{C}^{4}$ и рангом Чоя, равным 2 , для которых подпространство $\mathfrak{L}_{\theta}$ является некоммутативным графом смежности (см. [8; приложение]). Множество всех таких каналов обозначим $\widehat{\mathfrak{L}}_{\theta}$.

Для заданного $n \in \mathbb{N}$ пусть $\mathscr{H}_{n}$ - подпространство в $\mathbb{C}^{4 n}=\left[\mathbb{C}^{4}\right]^{\otimes n}$, порожденное векторами $x=e_{1} \otimes \cdots \otimes e_{1}+\mathrm{i} \cdot e_{2} \otimes \cdots \otimes e_{2}$ и $y=e_{3} \otimes \cdots \otimes e_{3}+\mathrm{i} \cdot e_{4} \otimes \cdots \otimes e_{4}$, где $\left\{e_{1}, \ldots, e_{4}\right\}-$ канонический базис в $\mathbb{C}^{4}$ (в каждом произведении $n$ сомножителей).

ТЕОрема. Пусть $\Phi_{\theta}-$ произвольный канал из $\widehat{\mathfrak{L}}_{\theta}$ и $n$ - любое натуралъное число.

А) У канала $\Phi_{\theta}$ есть идеальные подканаль тогда и толъко тогда, когда $\theta=\pi$.

Исследование выполнено за счет гранта Российского научного фонда (проект 14-21-00162).

DOI: $10.4213 / \mathrm{rm} 9642$ 
В) Если $\left|\theta_{1}\right|+\cdots+\left|\theta_{n}\right| \leqslant 2 \ln (3 / 2)$, то у канала $\Phi_{\theta_{1}} \otimes \cdots \otimes \Phi_{\theta_{n}}$ нет идеальных подканалов.

С) Если $\theta_{1}+\cdots+\theta_{n}=\pi(\bmod 2 \pi)$, то у канала $\Phi_{\theta_{1}} \otimes \cdots \otimes \Phi_{\theta_{n}}$ есть идеальный подканал, соответствующий подпространству $\mathscr{H}_{n}$.

Комбинируя утверждения В) и С) теоремы, получаем наш основной результат.

СледСтвиЕ 1. Пусть $n$ произвольно, а $m$ - такое натуральное число, что $\theta_{*}=$ $\pi / m \leqslant 2 \ln (3 / 2) / n$. Тогда у каналов $\Phi_{\theta_{*}}^{\otimes k}, k=1,2, \ldots, n$, нет идеальных подканалов, а у канала $\Phi_{\theta_{*}}^{\otimes m}$ есть идеалъный подканал, соответствующий подпространству $\mathscr{H}_{m}$.

В квантовой теории информации величина $\bar{Q}_{0}(\Phi)=\sup _{\mathscr{H} \in q_{0}(\Phi)} \log _{2} \operatorname{dim} \mathscr{H}$, где $q_{0}(\Phi)$ - множество всех подпространств в $\mathscr{H}_{A}$, на которых канал $\Phi$ обратим, называется одношаговой (one-shot) квантовой пропускной способностью канала $\Phi$ при нулевой ошибке. Эта величина характеризует предельную скорость безошибочной передачи квантовой информации при отсутствии блочного кодирования. Соответственно, (асимптотическая) квантовая пропускная способностью канала $\Phi$ при нулевой ошибке определяется с помощью регуляризации: $Q_{0}(\Phi)=\sup _{n} n^{-1} \bar{Q}_{0}\left(\Phi^{\otimes n}\right)$ [1], [2].

Следствие 1 показывает, что для любого $n$ существует такой канал $\Phi_{n}$ с $\mathscr{H}_{A}=\mathbb{C}^{4}$ и рангом Чоя 2 , что

$$
\bar{Q}_{0}\left(\Phi_{n}^{\otimes n}\right)=0, \quad \text { но } \quad Q_{0}\left(\Phi_{n}\right) \geqslant\left(\left[\frac{\pi n}{2 \ln (3 / 2)}\right]+1\right)^{-1}=\frac{2 \ln (3 / 2)}{\pi n}+o\left(\frac{1}{n}\right), \quad n \rightarrow+\infty,
$$

где $[x]$ - целая часть $x$.

Аналогичный результат для квантовой пропускной способности с $\varepsilon$-малой ошибкой был недавно получен в [9].

В силу утверждений А) и С) теоремы для каналов $\Phi_{\theta}$ имеет место суперактивация одношаговой квантовой пропускной способности при нулевой ошибке.

СледСтвие 2. Если $\theta \neq 0, \pi$, то для любых каналов $\Phi_{\theta} \in \widehat{\mathfrak{L}}_{\theta} u \Phi_{\pi-\theta} \in \widehat{\mathfrak{L}}_{\pi-\theta}$

$$
\bar{Q}_{0}\left(\Phi_{\theta}\right)=\bar{Q}_{0}\left(\Phi_{\pi-\theta}\right)=0, \quad \text { но } \quad \bar{Q}_{0}\left(\Phi_{\theta} \otimes \Phi_{\pi-\theta}\right)>0 .
$$

При любом $\theta \in(-\pi, \pi]$ канал $\Phi_{\theta} \otimes \Phi_{\pi-\theta}$ обратим на подпространстве $\mathscr{H}_{2}$.

В частности, канал $\Phi_{\pi / 2}$ дает пример симметричной суперактивации одношаговой квантовой пропускной способности при нулевой ошибке с рангом Чоя 2. Ранее такой пример с рангом Чоя 3 и тем же входным пространством $\mathscr{H}_{A}=\mathbb{C}^{4}$ был построен в [8].

Доказательство теоремы и ее многомерное обобщение приведены в [10].

\section{Список литературы}

[1] R. A. C. Medeiros, F. M. de Assis, Int. J. Quantum Inf., 3:1 (2005), 135-139. [2] R. Duan, S. Severini, A. Winter, IEEE Trans. Inform. Theory, 59:2 (2013), 1164-1174. [3] G. Smith, J. Yard, Science, 321:5897 (2008), 1812-1815. [4] T. S. Cubitt, J. Chen, A. W. Harrow, IEEE Trans. Inform. Theory, 57:12 (2011), 8114-8126. [5] R. Duan, Super-activation of zero-error capacity of noisy quantum channels, arXiv: 0906.2527. [6] А. С. Холево, Квантовые системъ, каналь, информачия, МЦНМО, М., 2010, 328 с. [7] Н. Ю. Решетихин, Л.А. Тахтаджян, Л. Д. Фаддеев, Алгебра и анализ, 1:1 (1989), 178-206. [8] М. Е. Широков, Т. В. Шульман, Пробл. передачи информ., 50:3 (2014), 35-50. [9] T. Cubitt, D. Elkouss, W. Matthews, M. Ozols, D. Perez-Garcia, S. Strelchuk, Unbounded number of channel uses are required to see quantum capacity, arXiv: 1408.5115. [10] M. E. Shirokov, On channels with positive quantum zero-error capacity having vanishing $n$-shot capacity, arXiv: 1407.8524 .

\section{Максим Евгеньевич Широков}

(Maxim E. Shirokov)

Математический институт им. В. А. Стеклова РАН

E-mail: msh@mi.ras.ru
Представлено А. В. Булинским Принято редколлегией 05.12 .2014 